\title{
Surface Treatment of Materials for Variable Applications and Surface Properties and Cha- racterization
}

Zdenka Kolska ${ }^{1}$, Monika Benkocka ${ }^{1}$, Tereza Knapova ${ }^{1}$, Nikola Slepickova Kasalkova ${ }^{2}$, Katerina Kolarova ${ }^{2}$, Petr Slepicka $^{2}$, Vaclav Svorcik ${ }^{2}$

${ }^{1}$ Faculty of Science, J. E. Purkyne University in Usti nad Labem. Ceske Mladeze 8, 40096 Usti nad Labem. Czech Republic. E-mail: zdenka.kolska@ujep.cz, benkockamonika@ @eznam.cz, tknapova@ seznam.cz

${ }^{2}$ Department of Solid State Engineering, University of Chemistry and Technology, Technická 3, 16628 Prague, Czech Republic. E-mail: Vaclav.svorcik@vscht.cz, nikola.kasalkova@vscht.cz, katerina.kolarova@vscht.cz, petr.slepicka@vscht.cz

Many materials of general interest, which they are frequently employed in common life, industry, biotechnology, tissue engineering studies, medicine, have many excellent properties, but their inert nature may limit their wider usage in some cases. Many modification techniques have been therefore developed to improve surface properties of variable substrates for other applications. For a long time we study surface properties of a wide range of substrates (polymers, glass, silicate powders, etc.). Surfaces of studied materials are firstly activated by chemical or physical ways and than variable chemical compounds (e.g. nanoparticles, nanostructures or nanolyers) are grafted or deposited on. Surface properties are characterized before and after individual activation or modification steps by many techniques (microscopies, spectroscopies, goniometry, electrokinetic analyses, X-ray diffraction, etc.). Some of tested samples are also tested for antimicrobial activities and/or for cell adhesion and proliferation for their potential usage in tissue engineering. This paper presents a survey of techniques for preparation of nanostructured materials for usage in electronic, optics or medicine and selected results.

Keywords: Surface Modification, Surface Properties, Surface Characterization, Surface Activation, Grafting

\section{Acknowledgement}

This work was supported by the Czech Science Foundation (GA CR) under the project no. 13-06609S, by the Grant Agency of Health Ministry under project no. 15-33018A and by the internal grants of the J. E. Purkinje University in Usti nad Labem in the Czech Republic (SGA projects no. 5322215001401 and 5351815000801). The authors acknowledge the assistance provided by the Research Infrastructure NanoEnviCz, supported by the Ministry of Education, Youth and Sports of the Czech Republic under project No. LM2015073.

\section{References}

[1] BAČÁKOVÁ, L., FÍLOVÁ, E., PAŘíZEK, M., RUML, T., ŠVORČÍK, V. (2011). Control of cell adhesion, proliferation and differentiation on materials designed for body implants. Biotechnology Advances, 29, pp. 739-767.

[2] REZNICKOVA, A., SIEGEL, J., SLAVIKOVA, N., KOLSKA, Z., STASZEK, M., SVORCIK, V. (2016). Metal nanoparticles designed PET: preparation, characterization and biological response. Reactive and Functional Polymers, 105 , pp. 1-8.

[3] KOLSKÁ, Z., ŘEZNíČKOVÁ, A., NAGYOVÁ, M., SLEPIČKOVÁ KASÁlKOVÁ, N., SAJDL, P., SLEPIČKA, P., ŠVORČÍK, V. (2014). Plasma activated polymers grafted with cysteamine for bio-application. Polymer Degradation and Stability, 101, pp. 1-9.

[4] LUPÍNKOVÁ, S., VÝBORNÝ, K., BENKOCKÁ, M., KOLSKÁ, Z., SLEPIČKOVÁ KASÁLKOVÁ, N., ŠVORČÍK, V. (2014). Analýza povrchů polymerů aktivovaných plazmatem a následně roubovaných vicinálními sloučeninami. Chemické Listy, 108, pp. s237-s240.

[5] MIKUlÍKOVÁ, R., MORITZ, S., GUMPENBERGER, T., OLBRICH, M., ROMANIN, C., BAČÁKOVÁ, L., ŠVORČÍK, V., HEITZ, J. (2005). Cell microarrays on photochemically modified PTFE. Biomaterials, 26, pp. 5572-5580.

[6] SLEPIČKA, P., SLEPIČKOVÁ KASÁLKOVÁ, N., SIEGEL, J., KOLSKÁ, Z., BAČÁKOVÁ, L., ŠVORČÍK, V. (2015). Nano-structured and functionalized surfaces for cytocompatibility improvement and bactericidal action. Biotechnology Advances, 33, pp. 1120-1129.

[7] LI, L., HONG, M., SCHMIDT, M., ZHONG, M., MALSHE, A., HUIS, P., VELD, B. (2011). Laser nano-manufacturing - State of the art and challenges, CIRP Annals. Manufacturing Technology, 11, pp. 735-55.

[8] SVOBODOVÁ, J., KRAUS, P. (2015). Influence of Chemical Pre-treatments Nanotechnology Based Applied to the Al Sheet on the Roughness and Morphology of the Surface. Manufacturing Technology, 15, pp. 714-720.

[9] DOAN, V.T., KUSMIC, D., POSPICHAL, M. (2015). Surface Treatment Technologies for Wear Resistance Increasing of 42CrMo4 Steel. Manufacturing Technology, 15, pp. 303-307. 
[10] SLEPIČKA, P., CHALOUPKA, A., SAJDL, P., HEITZ, J., HNATOWICZ, V., ŠVORČÍK, V. (2011). Angle dependent laser nanopatterning of poly(ethylene terephthalate) surfaces. Applied Surface Science, 257, pp. 60216025 .

[11] SIEGEL, J., HEITZ, J., ŘEZNÍČKOVÁ, A., ŠVORČÍK, V. (2013). Preparation and characterization of fully separated gold nanowire arrays. Applied Surface Science, 264, pp. 443-447.

[12] SLEPIČKA, P., NEDĚLA, O., SIEGEL, J., KRAJCAR, R., KOLSKÁ, Z., ŠVORČÍK, V. (2014). Ripple polystyrene nano-pattern induced by KrF laser. EXPRESS Polymer Letters, 8, pp. 459-466.

[13] SLEPIČKA, P., NEDĚLA, O., SAJDL, P., KOLSKÁ, Z., ŠVORČÍK, V. (2013). Polyethylenenaphtalate as an excellent candidate for ripple nanopatterning. Applied Surface Science, 285B, pp. 885-892.

[14] ZAKOVA, P., KASALKOVA SLEPICKOVA, N., KOLSKA, Z., LEITNER, J., KARPISKOVA, J., STIBOR, I., SLEPICKA, P., SVORCIK, V. (2016). Cytocompatibility of amine functionalized carbon nanoparticles grafted on polyethylene. Materials Science \& Engineering C-Materials for Biological Applications, 60, pp. 394-401.

[15] ŘEZNÍČKOVÁ, A., KOLSKÁ, Z., SIEGEL, J., ŠVORČÍK, V. (2012). Grafting of gold nanoparticles and nanorods on plasma-treated polymers by thiols. Journal of Material Science, 47, pp. 6297-6304.

[16] ŠVORČÍK, V., KOLSKÁ, Z., KVÍTEK, O., SIEGEL, J., ŘEZNÍČKOVÁ, A., ŘEZANKA, P., ZÁRUBA, K. (2011). "Soft and rigid" dithiols and Au nanoparticles grafting on plasma-treated polyethyleneterephthalate. $N a$ noscale Research Letters, 6, pp. 607-613.

[17] ŠVORČÍK, V., KOLSKÁ, Z., SIEGEL, J., SLEPIČKA, P. (2013). "Short” Dithiol and Au Nanoparticles Grafting on Plasma Treated Polyethyleneterephthalate. Journal of Nano Research, 25, pp. 40-48.

[18] ŘEZNÍČKOVÁ, A., KOLSKÁ, Z., ZÁRUBA, K., ŠVORČÍK, V. (2014). Grafting of gold nanoparticles on polyethyleneterephthalate using dithiol interlayer. Materials Chemistry and Physics, 145, pp. 484-490.

[19] ŠVORČÍK, V., MAKAJOVÁ, Z., KASÁLKOVÁ, N., KOLSKÁ, Z., BAČÁKOVÁ, L. (2012). Plasma-Modified and Polyethylene Glycol-Grafted Polymers for Potential Tissue Engineering Applications. Journal of Nanoscience and Nanotechnology, 12, pp. 6665-6671.

[20] KOLSKÁ, Z., MATOUŠEK, J., ČAPKOVÁ, P., BRABOREC, B., ČERNÁ, H., BENKOCKÁ, M., LONDESBOROUGH, M.G.S. (2015). A luminescent montmorillonite/borane nanocomposite. Applied Clay Science, 118, pp. 295-300.

[21] LUPÍNKOVÁ, S., BENKOCKÁ, M., BRABOREC， J., MATOUŠEK, J., KOLÁŘOVÁ, K., LONDESBOROUGH, M. G. S., KOLSKÁ, Z. (2016). Analýza chemicky modifikovaných polymerních povrchů. Czech Chemical Society Symposium Series, 14, pp. 19-22.

[22] KOLSKÁ, Z., MAKAJOVÁ, Z., KOLÁŘOVÁ, K., KASÁlKOVÁ SLEPIČKOVÁ, N., TROSTOVÁ, S., ŘEZNÍČKOVÁ, A., SIEGEL, J., ŠVORČÍK, V. (2013). Electrokinetic potential and other surface properties of polymer foils and their modifications. In: Polymer Science, pp. 203-228. In Tech d.o.o., Rijeka, Croatia.

[23] KOLSKÁ, Z., ŘEZNÍČKOVÁ, A., ŠVORČÍK, V. (2012). Surface characterization of polymer foils. $e$-Polymers, Article No. 083, pp. 1-13.

[24] KOLSKÁ, Z., SLEPIČKOVÁ KASÁLKOVÁ, N., SIEGEL, J., ŠVORČÍK, V. (2013). Electrokinetic Potential for Characterization of Nanosctructured Solid Flat Surfaces. Journal of Nano Research, 25, pp. 31-39.

[25] HIEMENZ, P., RAJAGOPALAN, R. (1997). The electrical double layer and double-layer interactions. In: Principles of colloid and surface chemistry, pp. 499-533, Marcel Dekker, Inc., New York.

[26] BEATTIE, J. K. (2006). The intrinsic charge on hydrophobic microfluidic substrates. Laboratory Chip, 6, pp. 1409-1411.

[27] KOSMULSKI, M. (2001). Chemical properties of material surfaces. 102, Marcel Dekker, Inc., New York, 2001.

[28] KlikA, Z., PUSTKOVÁ, P., PRAUS, P., KOVÁŘ, P., POSPIŠIL, M., MALÝ, P., GRYGAR, T., KULHÁNKOVÁ, L., ČAPKOVÁ, P. (2009). Fluorescence of reduced charge montmorillonite complexes with methylene blue: Experiments and molecular modeling. Journal of Colloid and Interface Science, 339, pp. 416423.

[29] KlikA, Z., PUSTKOVÁ, P., DUdOVÁ, M., ČAPKOVÁ, P., KLIKOVÁ, C., GRYGAR, T.M. (2011). The adsorption of methylene blue on montmorillonite from acid solutions. Clay Minerals, 46, pp. 461-471.

[30] POLIVKOVA, M., SIEGEL, J., KOLAROVA, K., RIMPELOVA, S., SVORCIK, V. (2016). Silver Nanostructures Sputtered on Polyimide: Surface Characterization and Antibacterial Properties. Chemické Listy, 110, pp. 139143.

[31] SIEGEL, J., POLIVKOVA, M., STASZEK, M., KOLAROVA, K., RIMPELOVA, S., SVORCIK, V. (2015). Nanostructured silver coatings on polyimide and their antibacterial response. Materials Letters, 145, pp. 87-90. 